\title{
Designing of Repetitive Group Sampling Plan under the Inverse Gaussian Distribution
}

\author{
G. Kannan, S. Balamurali
}

\begin{abstract}
This study investigates the attributes repetitive group sampling plans based on a truncated life test under the inverse Gaussian distribution with known shape parameter. The sample size and two acceptance numbers are the three design parameters determined for the proposed repetitive group sampling plans for different mean ratios. Tables are constructed to determine the optimal design parameters for different values of shape parameters of the inverse Gaussian model and the results are explained by with examples. Also the effect of misspecification of shape parameters is also discussed.
\end{abstract}

Keywords: Consumer's risks, Inverse Gaussian distribution, Producer's risks, Repetitive group sampling plans, Truncated life test.

\section{INTRODUCTION}

Acceptance sampling is an essential part of statistical quality control that allows industries to check the quality of products. A company cannot check every one of its products produced. Acceptance sampling solves this by inspecting only a sample of the items taken from the lot. The acceptance sampling is a testing procedure that involves taking random samples from lot of goods and exploring them for particular needs. An acceptance sampling plan recommends the sample size to be examined and acceptance criteria for the judgment of the lot by the inspection of the sample of the items. This will reduce the inspection cost and time. In acceptance sampling, decisions are made using random sample results, so there may be a chance to reject the good quality lot or to accept the poor quality lot. The producer will be affected when the good lot is rejected and the consumer will be affected when the unqualified lot or bad lot is accepted. Therefore, the probability of rejecting a good lot and the probability of accepting a bad lot are usually defined as the producer's risk $(\alpha)$ and consumer's risk $(\beta)$, respectively. The quality levels corresponding to the producer's risk and consumer's risk are respectively termed as producer quality level (PQL) and consumer quality level (CQL).

The concept of time-truncated life test is earning more admiration in the acceptance sampling. Typically, a life test is performed to examine the lifetime of the goods under a specified time, and this test is used to provide a life time warranty. Sampling plan based on time truncated life tests have been used to study the trustability of the goods. In many situations, the quality of goods is calculated through the

Revised Manuscript Received on December 15, 2019.

G. Kannan, Department of Mathematics, Kalasalingam Academy of Research and Education, Krishnankoil - 626126, Tamilnadu, India. Email: mr.kannan1691@gmail.com.

S. Balamurali*, Department of Computer Applications, Kalasalingam Academy of Research and Education, Krishnankoil - 626126, Tamilnadu, India. Email: sbmurali@rediffmail.com lifetime $(T)$ and the scale parameter of the distribution of $T$. If we take $\mu$ is the true mean lifetime and $\mu_{0}$ is the specified mean lifetime of the products, then the ratio $\mu / \mu_{0}$ is considered as the quality of level of item. Therefore, in time truncated life test schemes, we must determine the lowest values of $n$, the sample size to ensure the mean lifetime. Also, the life test is stopped either when pre-assigned time is reached or if the number of failures exceeds the given acceptance number $c$.

Several authors have investigated the life test sampling plans under various lifetime distributions. A study on acceptance sampling plan based on time truncated life tests under exponential distribution has done by [1]. The sampling plans to assure the Weibull distributed lifetime when the shape parameter is known have been designed by [2]. Under gamma distribution with known shape parameter, the sampling plans have been proposed by [3]. Acceptance sampling plans based on truncated life tests under the Birnbaum-Saunders model have been proposed by [4]. Acceptance sampling plan to assure the quality of the products where the lifetime follows exponentiated inverse Rayleigh distribution have been discussed by [5]. Acceptance sampling plan based on truncated life test using Rayleigh distribution studied by [6]. In [7], the authors designed the group acceptance sampling plan under Weibull distribution based on truncated life test. Similarly, group acceptance sampling plans have been developed by [8] under the inverse Rayleigh distribution and log-logistic distribution.

\section{INVERSE GAUSSIAN DISTRIBUTION}

Generally, inverse Gaussian (IG) distribution is considered as the most powerful distribution in statistics and the applications of this distribution can be seen in different fields (see for example, [9], [10]). This distribution also has been used as a model to express the lifetime by [11]. In order to monitor the process centrality and dispersion, control chart has been designed by [12] used IG distribution. Sequential sampling plan has been proposed by [13] using this distribution. In [14], the authors developed reliability sampling plans for truncated life tests with IG life time data. The group sampling plan under IG distribution has been proposed by [15]. IG distribution is a good choice among the popularized lifetime distributions such as Weibull, gamma and log-normal. The distribution has a broad usage area in clinical experiment, standard and reliability or trustability theory, industrial engineering applications and life tests. The cumulative distribution function (cdf) of IG distribution is given by 


$$
F(t)=\Phi\left\{\sqrt{\frac{\delta \omega}{t}}\left(\frac{t}{\omega}-1\right)\right\}+\exp \{2 \delta\} \Phi\left\{-\sqrt{\frac{\delta \omega}{t}}\left(\frac{t}{\omega}+1\right)\right\}
$$

where $\delta>0$ is a shape parameter, $\omega>0$ is a scale parameter and $\Phi($.$) is the cdf of the standard normal distribution. For$ $m>0$, the $m^{\text {th }}$ moment of a random variable $T$ following IG distribution is given as

$$
E\left(T^{m}\right)=T^{m} \sum_{i=0}^{m-1} \frac{(m-1+i) !}{(m-1-i) !}\left(\frac{1}{2 \delta}\right)^{i}
$$

Especially, the first moment is given by

$$
E(T)=\mu=\omega
$$

\section{THE REPETITIVE GROUP SAMPLING PLAN}

Repetitive group sampling (RGS) plan was introduced by [16] and this sampling plan is frequently known as one of the special purpose sampling plans. According to [16], the operation of this proposed plan is similar to the sequential sampling plan, and it is more efficient than the single sampling plan (SSP) but less efficient than the sequential sampling plan. Many authors have developed different sampling plans using life testing concept under IG distribution. But none has designed the RGS plan for assuring mean lifetime of the products under IG distribution. In this paper, we explore the RGS plan for time truncated life test under the IG distribution when the shape parameter is known. Let $\mu$ be the true mean lifetime and let $\mu_{0}$ be the specified mean lifetime of the goods. To make the decision about the submitted goods, the ratio of the true mean life between the specified mean life of the goods are very helpful. A bad lot means that $\mu$ is below $\mu_{0}$ that is $\mu<\mu_{0}$. Otherwise, the lot is good. The implementation procedure of the proposed sampling plan is as follows.

Step 1:Take a random sample of size $n(\geq 1)$ items and put them on test for time $t$. If the number of failures $d$ is less than or equal to $c_{1}$, then accept the lot.

Step 2:Reject the lot if the number of failures is larger than $c_{2}$

Step 3:If $c_{1}<d \leq c_{2}$, then repeat Step 1 .

The proposed plan contains three parameters namely $n, c_{1}, c_{2}$. When $c_{1}=c_{2}$, the proposed plan reduces to SSP. When the lot size is adequate and large, it is well known that the binomial distribution is used. The probability of lot acceptance based on single sample under binomial distribution is given by

$$
P_{a}=P\left\{d \leq c_{1}\right\}=\sum_{d=0}^{c_{1}}\left(\begin{array}{l}
n \\
d
\end{array}\right) p^{d}(1-p)^{n-d}
$$

The rejection of the lot based on single sample under binomial distribution is given by

$$
P_{r}=P\left\{d>c_{2}\right\}=1-P\left\{d \leq c_{2}\right\}=1-\sum_{d=0}^{c_{2}}\left(\begin{array}{l}
n \\
d
\end{array}\right) p^{d}(1-p)^{n-d}
$$

The probability of repeated sampling $R$ is given by

$R=1-P_{a}-P_{r}$, Therefore

$$
\mathbf{1}-\boldsymbol{R}=\boldsymbol{P}_{a}+\boldsymbol{P}_{r}
$$

The operating characteristic (OC) function based on the proposed plan under binomial distribution is given as follows (see [16]).

$$
P_{a}(p)=\frac{P_{a}}{1-R}=\frac{P_{a}}{P_{a}+P_{r}}
$$

$$
P_{a}(p)=\frac{\sum_{d=0}^{c_{1}}\left(\begin{array}{l}
n \\
d
\end{array}\right) p^{d}(1-p)^{n-d}}{1-\sum_{d=0}^{c_{2}}\left(\begin{array}{l}
n \\
d
\end{array}\right) p^{d}(1-p)^{n-d}+\sum_{d=0}^{c_{1}}\left(\begin{array}{l}
n \\
d
\end{array}\right) p^{d}(1-p)^{n-d}}
$$

The Average Sample Number (ASN) of the proposed plan is given by

$$
A S N=\frac{n}{P_{a}+P_{r}}
$$

The probability that an item fails before the time $t_{0}$ is denoted by $p$ and is given as $p=F\left(t_{0}\right)$. It is to be noted that the cdf of the given in equation (1) depends on unknown $\omega$ only through the ratio $t / \omega$. To acquire $p$, it would be suitable to specify the termination time as a multiple of the specified lifetime $\mu_{0}$. If $t_{0}=a \mu_{0}$ where ' $a$ ' is a constant and called as experiment termination ratio, then the cdf of the given equation can also be written as

$$
\begin{gathered}
p=F\left(t_{0}\right) \\
F\left(t_{0}\right)=\Phi\left\{\sqrt{\frac{\delta}{a\left(\mu_{0} / \mu\right)}}\left(\frac{a}{\mu / \mu_{0}}-1\right)\right\}+\exp \{2 \delta\} \Phi\left\{-\sqrt{\frac{\delta}{a\left(\mu_{0} / \mu\right)}}\left(\frac{a}{\mu / \mu_{0}}+1\right)\right\}
\end{gathered}
$$

A well designed plan is one which minimizes both the producer's risk and the consumer's risk simultaneously. As pointed out by [7], the consumers desire that the lot acceptance probability to be lesser than their specified risk when $\mu=\mu_{0}$. The simultaneous consideration of following two inequalities is essential for finding the design parameters.

$$
\begin{aligned}
& P_{a}\left(p \mid \mu / \mu_{0}=r_{2}\right) \geq 1-\alpha \\
& P_{a}\left(p \mid \mu / \mu_{0}=1=r_{1}\right) \leq \beta
\end{aligned}
$$

It is noted that $r_{2}$ and $r_{1}$ are the mean ratios of the producer's risk and consumer's risk respectively. Let $p_{1}$ and $p_{2}$ be the failure probability equivalent to producer's risks and consumer's risks respectively. Then the design parameters are determined by solving the following optimization problem.

Minimize $\operatorname{ASN}\left(p_{1}\right)$

Subject to

$$
\begin{aligned}
& P_{a}\left(p_{1}\right) \geq 1-\alpha \\
& P_{a}\left(p_{2}\right) \leq \beta
\end{aligned}
$$

where

$$
\begin{gathered}
P_{a}\left(p_{1}\right)=\frac{\sum_{d=0}^{c_{1}}\left(\begin{array}{l}
n \\
d
\end{array}\right) p_{1}{ }^{d}\left(1-p_{1}\right)^{n-d}}{1-\sum_{d=0}^{c_{2}}\left(\begin{array}{l}
n \\
d
\end{array}\right) p_{1}{ }^{d}\left(1-p_{1}\right)^{n-d}+\sum_{d=0}^{c_{1}}\left(\begin{array}{l}
n \\
d
\end{array}\right) p_{1}{ }^{d}\left(1-p_{1}\right)^{n-d}} \\
P_{a}\left(p_{2}\right)=\frac{\sum_{d=0}^{c_{1}}\left(\begin{array}{l}
n \\
d
\end{array}\right) p_{2}{ }^{d}\left(1-p_{2}\right)^{n-d}}{1-\sum_{d=0}^{c_{2}}\left(\begin{array}{l}
n \\
d
\end{array}\right) p_{2}{ }^{d}\left(1-p_{2}\right)^{n-d}+\sum_{d=0}^{c_{1}}\left(\begin{array}{l}
n \\
d
\end{array}\right) p_{2}{ }^{d}\left(1-p_{2}\right)^{n-d}}
\end{gathered}
$$

Tables I - IV report the design parameters of the proposed plan for the known shape parameter values of IG distribution such as $\delta=2,3$, four values of consumer's risk $\beta=0.25,0.10$, $0.05,0.01$, two values of termination ratio $a=0.5,1.0$, 
and five values of mean ratio $r_{2}=2,2.5,3,3.5,4$. The producer's risk is considered as 0.05 (i.e., $5 \%$ ) for all tables. From the tables, it is observed that if the value of mean ratio increases,

then there are decreasing trends in acceptance number and sample size. We monitor the same behaviour in the design parameters as the experiment termination ratio increases. One can observe that there is no important change in design parameters when shape parameter increases while keeping other values such as $\beta, r_{2}$ and $a$ are constant

Table- I: Proposed plan for IG distribution with $\delta=2$ when $a=0.5$

\begin{tabular}{c|c|c|c|c|c|c|c|}
\hline$\beta$ & $r_{2}$ & $n$ & $c_{1}$ & $c_{2}$ & $P_{a}\left(p_{1}\right)$ & $P_{a}\left(p_{2}\right)$ & $A S N$ \\
\hline 0.25 & 2 & 7 & 0 & 1 & 0.9819 & 0.2354 & 8.388 \\
& 2.5 & 7 & 0 & 1 & 0.9979 & $\uparrow$ & 7.481 \\
& 3 & $\uparrow$ & $\uparrow$ & $\uparrow$ & 0.9997 & $\uparrow$ & 7.166 \\
& 3.5 & $\uparrow$ & $\uparrow$ & $\uparrow$ & 0.9999 & $\uparrow$ & 7.058 \\
& 4 & $\uparrow$ & $\uparrow$ & $\uparrow$ & 0.9999 & $\uparrow$ & 7.020 \\
0.10 & 2 & 10 & 0 & 1 & 0.9610 & 0.0905 & 12.774 \\
& 2.5 & 10 & 0 & 1 & 0.9955 & $\uparrow$ & 10.979 \\
& 3 & $\uparrow$ & $\uparrow$ & $\uparrow$ & 0.9994 & $\uparrow$ & 10.340 \\
& 3.5 & $\uparrow$ & $\uparrow$ & $\uparrow$ & 0.9999 & $\uparrow$ & 10.119 \\
& 4 & $\uparrow$ & $\uparrow$ & $\uparrow$ & 0.9999 & $\uparrow$ & 10.041 \\
& & & & & & & \\
0.05 & 2 & 13 & 0 & 2 & 0.9926 & 0.0499 & 18.681 \\
& 2.5 & 1 & 0 & 1 & 0.9934 & 0.0493 & 13.407 \\
& 3 & $\uparrow$ & $\uparrow$ & $\uparrow$ & 0.9992 & $\uparrow$ & 12.490 \\
& 3.5 & $\uparrow$ & $\uparrow$ & $\uparrow$ & 0.9999 & $\uparrow$ & 12.171 \\
& 4 & $\uparrow$ & $\uparrow$ & $\uparrow$ & $\uparrow$ & $\uparrow$ & 12.060 \\
& & & & & & & \\
0.01 & 2 & 26 & 1 & 2 & 0.9592 & 0.0094 & 29.858 \\
& 2.5 & 18 & 0 & 1 & 0.9846 & 0.0089 & 21.138 \\
& 3 & $\uparrow$ & $\uparrow$ & $\uparrow$ & 0.9981 & $\uparrow$ & 19.102 \\
& 3.5 & $\uparrow$ & $\uparrow$ & $\uparrow$ & 0.9997 & $\uparrow$ & 18.385 \\
& 4 & $\uparrow$ & $\uparrow$ & $\uparrow$ & $\uparrow$ & $\uparrow$ & 18.135 \\
& & & & & & & \\
\hline
\end{tabular}

$\uparrow$ : Use the plan above

Table- II: Proposed plan for IG distribution with $\delta=2$ when $a=1.0$

\begin{tabular}{|c|c|c|c|c|c|c|c|}
\hline$\beta$ & $r_{2}$ & $n$ & $c_{1}$ & $c_{2}$ & $P_{a}\left(p_{1}\right)$ & $P_{a}\left(p_{2}\right)$ & $A S N$ \\
\hline 0.25 & 2 & 3 & 0 & 2 & 0.9730 & 0.1726 & 6.453 \\
& 2.5 & 4 & 1 & 2 & 0.9898 & 0.2212 & 4.368 \\
& 3 & 3 & 0 & 1 & 0.9765 & 0.0698 & 3.774 \\
& 3.5 & $\uparrow$ & $\uparrow$ & $\uparrow$ & 0.9924 & $\uparrow$ & 3.447 \\
& 4 & $\uparrow$ & $\uparrow$ & $\uparrow$ & 0.9974 & $\uparrow$ & 3.259 \\
& & & & & & & \\
0.10 & 2 & 6 & 1 & 3 & 0.9520 & 0.0468 & 9.912 \\
& 2.5 & 5 & 1 & 2 & 0.9761 & 0.0846 & 5.690 \\
& 3 & 3 & 0 & 1 & 0.9765 & 0.0698 & 3.774 \\
& 3.5 & $\uparrow$ & $\uparrow$ & $\uparrow$ & 0.9924 & $\uparrow$ & 3.447 \\
& 4 & $\uparrow$ & $\uparrow$ & $\uparrow$ & 0.9974 & $\uparrow$ & 3.259 \\
& & & & & & & \\
0.05 & 2 & 6 & 1 & 3 & 0.9520 & 0.0468 & 9.912 \\
& 2.5 & 4 & 0 & 2 & 0.9834 & 0.0353 & 7.108 \\
& 3 & 4 & 0 & 1 & 0.9528 & 0.0220 & 5.344 \\
& 3.5 & $\uparrow$ & $\uparrow$ & $\uparrow$ & 0.9846 & $\uparrow$ & 4.788 \\
& 4 & $\uparrow$ & $\uparrow$ & $\uparrow$ & 0.9949 & $\uparrow$ & 4.459 \\
& & & & & & & \\
0.01 & 2 & 8 & 1 & 4 & 0.9537 & 0.0080 & 18.494 \\
& 2.5 & 5 & 0 & 2 & 0.9579 & 0.0097 & 10.035 \\
& 3 & $\uparrow$ & $\uparrow$ & $\uparrow$ & 0.9928 & 0.0097 & 7.574 \\
& 3.5 & 5 & 0 & 1 & 0.9743 & 0.0076 & 6.219 \\
& 4 & $\uparrow$ & $\uparrow$ & $\uparrow$ & 0.9914 & $\uparrow$ & 5.715 \\
\hline
\end{tabular}

$\uparrow:$ Use the plan above
Table- III: Proposed plan for IG distribution with $\delta=2$ when $a=0.5$

\begin{tabular}{|c|c|c|c|c|c|c|c|}
\hline$\beta$ & $r_{2}$ & $n$ & $c_{1}$ & $c_{2}$ & $P_{a}\left(p_{1}\right)$ & $P_{a}\left(p_{2}\right)$ & $A S N$ \\
\hline 0.25 & 2 & 11 & 0 & 1 & 0.9966 & 0.2174 & 11.934 \\
& 2.5 & $\uparrow$ & $\uparrow$ & $\uparrow$ & 0.9998 & $\uparrow$ & 11.199 \\
& 3 & $\uparrow$ & $\uparrow$ & $\uparrow$ & 0.9997 & $\uparrow$ & 11.042 \\
& 3.5 & $\uparrow$ & $\uparrow$ & $\uparrow$ & $\uparrow$ & $\uparrow$ & 11.009 \\
& 4 & $\uparrow$ & $\uparrow$ & $\uparrow$ & 1.0000 & $\uparrow$ & 11.001 \\
0.10 & 2 & 15 & 0 & 1 & 0.9935 & 0.0954 & 16.733 \\
& 2.5 & $\uparrow$ & $\uparrow$ & $\uparrow$ & 0.9998 & $\uparrow$ & 15.371 \\
& 3 & $\uparrow$ & $\uparrow$ & $\uparrow$ & 0.9999 & $\uparrow$ & 15.079 \\
& 3.5 & $\uparrow$ & $\uparrow$ & $\uparrow$ & $\uparrow$ & $\uparrow$ & 15.017 \\
& 4 & $\uparrow$ & $\uparrow$ & $\uparrow$ & 1.0000 & $\uparrow$ & 15.003 \\
0.05 & 2 & 19 & 0 & 1 & 0.9893 & 0.0435 & 21.769 \\
& 2.5 & $\uparrow$ & $\uparrow$ & $\uparrow$ & 0.9995 & $\uparrow$ & 19.596 \\
& 3 & $\uparrow$ & $\uparrow$ & $\uparrow$ & 0.9999 & $\uparrow$ & 19.127 \\
& 3.5 & $\uparrow$ & $\uparrow$ & $\uparrow$ & $\uparrow$ & $\uparrow$ & 19.027 \\
& 4 & $\uparrow$ & $\uparrow$ & $\uparrow$ & 1.0000 & $\uparrow$ & 19.005 \\
0.01 & 2 & 27 & 0 & 1 & 0.9779 & 0.0099 & 32.527 \\
& 2.5 & $\uparrow$ & $\uparrow$ & $\uparrow$ & 0.9990 & $\uparrow$ & 28.203 \\
& 3 & $\uparrow$ & $\uparrow$ & $\uparrow$ & 0.9999 & $\uparrow$ & 27.257 \\
& 3.5 & $\uparrow$ & $\uparrow$ & $\uparrow$ & $\uparrow$ & $\uparrow$ & 27.055 \\
& 4 & $\uparrow$ & $\uparrow$ & $\uparrow$ & $\uparrow$ & $\uparrow$ & 27.011 \\
\hline
\end{tabular}

\section{A. Example}

An examiner wants to run a test for 500 hours and he or she knows that the lifetime of the product follows the IG distribution with $\delta=2$. The specified durability of the submitted product is 1000 hours and the termination ratio is 0.5 . It is assumed that $\alpha=0.05, \beta=0.05$ and $r_{2}=2$. Then the optimal design parameters are obtained as $n=13, c_{1}=0$ and $c_{2}=2$ from Table I. So, the sampling plan can be executed as follows: Take a random sample of 13 items from the current lot and put it on test for 500 hours. If the number of failures reaches 3 , discard the lot and stop the test. If there is no failure in 500 hours, accept the lot. Repeat the test if the number of failures is 1 or 2 .

Table- IV: Proposed plan for IG distribution with $\delta=2$ when $a=0.5$

\begin{tabular}{|c|c|c|c|c|c|c|c|}
\hline$\beta$ & $r_{2}$ & $n$ & $c_{1}$ & $c_{2}$ & $P_{a}\left(p_{1}\right)$ & $P_{a}\left(p_{2}\right)$ & $A S N$ \\
\hline 0.25 & 2 & 3 & 0 & 2 & 0.9933 & 0.2128 & 5.000 \\
& 2.5 & 3 & 0 & 1 & 0.9798 & 0.0842 & 3.721 \\
& 3 & $\uparrow$ & $\uparrow$ & $\uparrow$ & 0.9958 & $\uparrow$ & 3.330 \\
& 3.5 & $\uparrow$ & $\uparrow$ & $\uparrow$ & 0.9991 & $\uparrow$ & 3.151 \\
& 4 & $\uparrow$ & $\uparrow$ & $\uparrow$ & 0.9998 & $\uparrow$ & 3.069 \\
0.10 & 2 & 4 & 0 & 2 & 0.9728 & 0.0464 & 7.758 \\
& 2.5 & 3 & 0 & 1 & 0.9798 & 0.0842 & 3.372 \\
& 3 & $\uparrow$ & $\uparrow$ & $\uparrow$ & 0.9958 & $\uparrow$ & 3.330 \\
& 3.5 & $\uparrow$ & $\uparrow$ & $\uparrow$ & 0.9991 & $\uparrow$ & 3.151 \\
& 4 & $\uparrow$ & $\uparrow$ & $\uparrow$ & 0.9998 & $\uparrow$ & 3.069 \\
& & & & & & & \\
0.05 & 2 & 4 & 0 & 2 & 0.9728 & 0.0464 & 7.758 \\
& 2.5 & 4 & 0 & 1 & 0.9593 & 0.0278 & 5.255 \\
& 3 & $\uparrow$ & $\uparrow$ & $\uparrow$ & 0.9917 & $\uparrow$ & 4.584 \\
& 3.5 & $\uparrow$ & $\uparrow$ & $\uparrow$ & 0.9982 & $\uparrow$ & 4.269 \\
& 4 & $\uparrow$ & $\uparrow$ & $\uparrow$ & 0.9996 & $\uparrow$ & 4.124 \\
& & & & & & & \\
0.01 & 2 & 8 & 1 & 3 & 0.9606 & 0.0089 & 12.190 \\
& 2.5 & 8 & 1 & 2 & 0.9798 & 0.0078 & 8.887 \\
& 3 & 6 & 0 & 1 & 0.9790 & $\uparrow$ & 7.928 \\
& 3.5 & $\uparrow$ & $\uparrow$ & $\uparrow$ & 0.9956 & $\uparrow$ & 6.603 \\
& 4 & $\uparrow$ & $\uparrow$ & $\uparrow$ & 0.9990 & $\uparrow$ & 6.278 \\
\hline
\end{tabular}

$\uparrow:$ Use the plan above 


\section{COMPARATIVE STUDY}

In this section, the $A S N\left(p_{1}\right)$ of RGS plan and SSP under IG distribution based on truncated life test are compared when the shape parameter value is 2 . For this comparison, we consider the values of $\beta=0.25,0.10,0.05,0.01, a=0.5,1.0$ and mean ratios $2,2.5,3,3.5,4$. We determine $A S N\left(p_{1}\right)$ for SSP under IG distribution and the values of $A S N\left(p_{1}\right)$ for both plans are located in Table V. From this table, we can see that the RGS plan involves minimum $A S N\left(p_{1}\right)$ for all values of the mean ratios when compared to the SSP. For example, when the mean ratio is $2, \beta=0.05$ and the shape parameter is 2 , the $A S N\left(p_{1}\right)$ of the RGS plan and the SSP are 18.68 and 25 respectively. The same trend can be observed when $a=1.0$. It clearly indicates that the proposed RGS plan under IG distribution outperforms the existing SSP.

Table- V: ASN of RGS plan and SSP when $a=0.5$

\begin{tabular}{|c|c|c|c|}
\hline$\beta$ & $r_{2}$ & $R G S$ & $S S P$ \\
\hline \multirow[t]{5}{*}{0.25} & 2 & 8.38 & 11 \\
\hline & 2.5 & 7.48 & 11 \\
\hline & 3 & 7.16 & 11 \\
\hline & 3.5 & 7.05 & 11 \\
\hline & 4 & 7.02 & 11 \\
\hline \multirow[t]{5}{*}{0.10} & 2 & 12.77 & 22 \\
\hline & 2.5 & 10.97 & 16 \\
\hline & 3 & 10.34 & 16 \\
\hline & 3.5 & 10.11 & 16 \\
\hline & 4 & 10.04 & 16 \\
\hline \multirow[t]{5}{*}{0.05} & 2 & 18.68 & 25 \\
\hline & 2.5 & 13.40 & 19 \\
\hline & 3 & 12.49 & 19 \\
\hline & 3.5 & 12.17 & 19 \\
\hline & 4 & 12.06 & 19 \\
\hline \multirow[t]{5}{*}{0.01} & 2 & 29.85 & 40 \\
\hline & 2.5 & 21.13 & 26 \\
\hline & 3 & 19.10 & 26 \\
\hline & 3.5 & 18.38 & 26 \\
\hline & 4 & 18.13 & 26 \\
\hline
\end{tabular}

\section{EFFECT OF MISSPECIFICATION OF SHAPE PARAMETER}

In this study, the shape parameter of the IG distribution is assumed to be known. It may be of interest, therefore, to see the effect of misspecification of the shape parameter on the acceptance probability. Let us suppose assume that the true shape parameter is denoted by $\delta^{1}$, which is probably different from particular one $\delta$ utilized for designing the sampling plan. Let us take $p_{1}{ }^{1}$ and $p_{2}{ }^{1}$ be the PQL and CQL corresponding to the true shape parameter. Then,

$$
\begin{aligned}
& p_{1}{ }^{1}=\Phi\left\{\sqrt{\frac{\delta^{1}}{a\left(\mu_{0} / \mu\right)}}\left(\frac{a}{\mu / \mu_{0}}-1\right)\right\}+\exp \left\{2 \delta^{1}\right\} \Phi\left\{-\sqrt{\frac{\delta^{1}}{a\left(\mu_{0} / \mu\right.}}\left(\frac{a}{\mu / \mu_{0}}+1\right)\right\} . \\
& p_{2}{ }^{1}=\Phi\left\{\sqrt{\frac{\delta^{1}}{a}}(a-1)\right\}+\exp \left\{2 \delta^{1}\right\} \Phi\left\{\sqrt{\frac{\delta^{1}}{a}}(a+1)\right\} .
\end{aligned}
$$

Therefore, the respective probabilities of acceptance at PQL and CQL will be $P_{a}\left(p_{1}{ }^{1}\right)$ and $P_{a}\left(p_{2}{ }^{1}\right)$ based on Eq. (15) and (16). Suppose the parameters $\left(n, c_{1}, c_{2}\right)$ are determined for the specified shape parameter $\delta$. Then there may be no problems from the misspecification of the shape parameter if they still satisfy the producer's risk and the consumer's risk (i.e., $\left.P_{a}\left(p_{1}{ }^{1}\right) \geq 1-\alpha\right)$ and $\left.P_{a}\left(p_{2}{ }^{1}\right) \leq \beta\right)$.
When designing the proposed plan, we use the shape parameter $\delta=3$, but assume that the actual value is different from this. We want to know the effect of the misspecification of the shape parameter on the probability of accepting a lot at the actual value. Table VI shows the acceptance probabilities at PQL and CQL for the proposed sampling plan with $a=0.5$ if the actual shape parameter (assumed to be 2.5 and 3.5 ) is different from the one specified $(\delta=3)$. It is observed that producer's risk is satisfied even for smaller value of the shape parameter than the specified one except when $\beta=0.01$ and $r_{2}=2$. It is also seen that the consumer risks are satisfied only

\begin{tabular}{|c|c|c|c|c|c|}
\hline \multirow[b]{2}{*}{$\beta$} & \multirow[b]{2}{*}{$r_{2}$} & \multicolumn{2}{|c|}{$\delta=2.5$} & \multicolumn{2}{|c|}{$\delta=3.5$} \\
\hline & & $P_{a}\left(p_{1}\right)$ & $P_{a}\left(p_{2}\right)$ & $P_{a}\left(p_{1}\right)$ & $P_{a}\left(p_{2}\right)$ \\
\hline \multirow[t]{5}{*}{0.25} & 2 & 0.9875 & 0.1302 & 0.9990 & 0.3226 \\
\hline & 3 & 0.9999 & 0.1302 & 0.9999 & 0.3226 \\
\hline & 4 & 0.9999 & 0.1302 & 1.0000 & 0.3226 \\
\hline & 5 & 1.0000 & 0.1302 & 1.0000 & 0.3226 \\
\hline & 6 & 1.0000 & 0.1302 & 1.0000 & 0.3226 \\
\hline \multirow[t]{5}{*}{0.10} & 2 & 0.9760 & 0.0489 & 0.9981 & 0.1625 \\
\hline & 3 & 0.9998 & 0.0489 & 0.9981 & 0.1625 \\
\hline & 4 & 0.9999 & 0.0489 & 0.9999 & 0.1625 \\
\hline & 5 & 1.0000 & 0.0489 & 1.0000 & 0.1625 \\
\hline & 6 & 1.0000 & 0.0489 & 1.0000 & 0.1625 \\
\hline \multirow[t]{5}{*}{0.05} & 2 & 0.9608 & 0.0194 & 0.9970 & 0.0832 \\
\hline & 3 & 0.9997 & 0.0194 & 0.9587 & 0.0832 \\
\hline & 4 & 0.9999 & 0.0194 & 0.9587 & 0.0832 \\
\hline & 5 & 1.0000 & 0.0194 & 0.9587 & 0.0832 \\
\hline & 6 & 1.0000 & 0.0194 & 0.9587 & 0.0832 \\
\hline \multirow[t]{5}{*}{0.01} & 2 & 0.9197 & 0.0033 & 0.9938 & 0.02358 \\
\hline & 3 & 0.9995 & 0.0033 & 0.9999 & 0.02358 \\
\hline & 4 & 0.9999 & 0.0033 & 1.0000 & 0.02358 \\
\hline & 5 & 1.0000 & 0.0033 & 1.0000 & 0.02358 \\
\hline & 6 & 1.0000 & 0.0033 & 1.0000 & 0.02358 \\
\hline
\end{tabular}
when the actual parameter is smaller than the specified one.

\section{CONCLUSION}

In this paper, an attribute RGS plan based on truncated life tests has been proposed when the life time of the given product follows IG distribution with known shape parameter. The sample size and acceptance number of the proposed RGS plan have been determined by considering the mean ratios as the quality of the product and the proposed plan is compared with SSP to show the effectiveness of the proposed plan. Also, it has been investigated that the effect of misspecification of shape parameter on the lot acceptance probabilities at PQL and CQL. The proposed RGS plan is flexible and it outperforms the SSP with respect to ASN required. From the discussion, it is clear that RGS plan gives better results than SSP when the failure time of product follows IG distribution.

\section{ACKNOWLEDGMENT}

The authors would like to thank the editor and anonymous reviewers for their useful comments and suggestions, which significantly improve the presentation of this manuscript. 


\section{REFERENCES}

1. M. Sobel, and J. A. Tischendrof, "Acceptance sampling with new life test objectives," Proceedings of the Fifth National Symposium on Reliability and Quality Control, Philadelphia, USA, 1959.

2. H. P. Goode, and J. H. K. Kao, "Sampling plans based on Weibull distribution," Proceedings of the Seventh National Symposium on Reliability and Quality Control, Philadelphia, pp. 24-40, 1961.

3. S. S. Gupta, and P. A. Groll, "Gamma distribution in acceptance sampling based on life tests," J. Amer. Stat. Assoc., vol. 56, pp. 942-970, 1961.

4. A. Baklizi, and A. Masri, "Acceptance sampling based on truncated life tests in the Birnbaum Saunders model," Risk Anal., vol. 24, pp. 1453-1457, 2004.

5. G. V. Ramachandran, and M. Palanivel, “Acceptance sampling plan from truncated life tests based on exponentiated inverse Rayleigh distribution," Amer. J. Math. Manag. Sci., vol. 33, pp. 20-35, 2014.

6. M. Aslam, "Double acceptance sampling based on truncated life tests in Rayleigh distribution,” Eur. J. Sci. Res., vol. 17, pp. 605-611, 2007.

7. M. Aslam, and C. H. Jun, "A group acceptance sampling plan for truncated life test having Weibull distribution,” J. Appl. Stat., vol. 39, pp. 1021-1027, 2009.

8. M. Aslam, and C. H. Jun, "Group acceptance sampling plan for truncated life tests based on the inverse Rayleigh distribution and log logistic distribution," Pak. J. Stat., vol. 25, pp. 107-119, 2009.

9. E. Schrodinger, "Zur Theorie der Fall-und Steig Versuche an Teilchen mit Brownsche Bewegung," Phys. Z., vol. 16, pp. 289-295, 1915.

10. A. Lancaster, “A stochastic model for the duration of a strike," J. R. Stat. Soc., Vol. 135, pp. 257-271, 1972.

11. R. S. Chhikara, and J. L. Folks, "The inverse Gaussian distribution as a life time model," Technometrics, vol. 19, pp. 461-468, 1977.

12. R. L. Edgeman, "Inverse Gaussian control charts," Aust. J. Stat., vol.31, pp. 78-84, 1989.

13. R. L. Edgeman, and P. M. Salzberg, "A sequential sampling plan for the inverse Gaussian mean," Stat. Pap., vol. 32, pp. 45-53, 1991.

14. T. R. Tsai, and C. J. Wu, "Reliability sampling plans under truncated life tests with inverse Gaussian lifetime data," Biomed. Soft Comput. Hum. Sci., vol. 13, pp. 13-24, 2008.

15. M. Aslam, M. Azam, and C. H. Jun, "Decision rule based on group sampling plan under the inverse Gaussian distribution," Sequential Anal., vol. 32, pp. 71-82, 2013.

16. R. E. Sherman, "Design and evaluation of repetitive group sampling plan," Technometrics, vol. 7, pp. 11-21, 1965.

17. S.Balamurali and C-H Jun, "Repetitive group sampling plan procedure for variable inspection," J. Appl. Stat., vol. 33(3), pp. 327-338, 2006.

18. M.Aslam, C-W.Wu, C-H Jun, M.Azam, N.Itay, "Developing a variables repetitive group sampling plan based on process capability index $C_{p k}$ with known mean and variance," J.Stat.Comput.Simul., vol. 83, pp.1507-1517, 2013.

19. A.yan, S.Liu, Dong, Xiaojuan, "Repetitive group sampling plan for Rayleigh products based on the lifetime performance index $C L$," J.Comput.Theor.Nanos, vol. 12, pp. 3631-3636, 2015.

20. A.yan and S.Liu, "Designing a repetitive group sampling plan for Weibull distributed process," Math.Probl.Eng, vol. 2016, pp. 10, 2016.

21. Balamurali.S and Usha.M, "A new repetitive group sampling plan based on the process capability index for product acceptance," Int.J.Qual.Reliab.Manag, vol. 35(2), pp. 463-480, 2018.

\section{AUTHORS PROFILE}

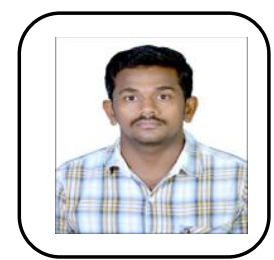

G. Kannan completed M. Sc. and M. Phil. in Mathematics at V.H.N.S.N. College, Virudhunagar, Tamilnadu. He is pursuing $\mathrm{Ph} . \mathrm{D}$ in the department of mathematics at Kalsaslingam Academy of Research and Education, Krishnankoil, Tamil Nadu since 2018. His current research interests include Acceptance sampling plan.

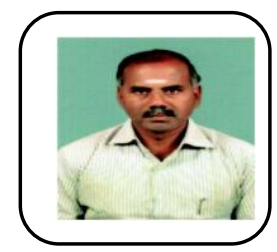

S. Balamurali is a professor of Statistics at Kalasalingam University. He earned his M.Sc. and Ph.D. degrees from Bharathiyar University, India. His research interests include statistical process control, acceptance sampling and analysis of means. 